\title{
On Board Accumulator Model for Power Flow Studies in DC Traction Networks
}

\author{
Pablo Arboleyaa ${ }^{\mathrm{a}, *}$, Manuel Coto ${ }^{\mathrm{a}}$, Cristina González-Morán ${ }^{\mathrm{a}}$, Ruth Arreguib \\ ${ }^{a}$ Department of Electrical Engineering, University of Oviedo, Spain \\ ${ }^{b} C A F I+D$ S.L.
}

\begin{abstract}
This paper presents a detailed model of a DC train to be applied to an unified AC/DC power flow analysis. The model will consider the regenerative braking of the train and the squeezing control that derives part or all regenerated power to the rheostatic braking system depending on the catenary voltage. The model also considers an on board accumulator (ACR) that can be charged with the regenerated power, depending on the network and train parameters. When the train is in traction mode, both the network and the ACR can contribute in feeding the train traction system.
\end{abstract}

(C) 2013 Published by Elsevier Ltd.

Keywords:

Graph theory, Rail transportation power systems, AC/DC power flow, On-board Accumulator

\section{Introduction}

There exist many works modeling DC traction systems and the AC systems that feed the traction one [1]. Some approaches use the sequential method applying an iterative procedure between the AC system and the DC system. In such cases an initial voltage profile in the DC system is assumed, then the DC power flow is solved calculating the power extracted from the AC system and finally, the AC power flow is used to correct the initial DC voltage profile [2-4]. Other authors prefer the use of unified methods also called extended variable methods, that solve the whole equations system [5, 6]. In [7], a deep study of these methods is presented and a new implementation for solving the AC/DC power flow, by using the unified method approach is done. This method uses a matrix formulation that facilitates the way in which the problem is set out. This work is focused on the network modeling, and even when some components such as converters, different type of buses and trains are modeled, the models used are quite simplistic. For instance, the trains are considered as fixed active power loads. At each instant the power value is provided by another complex software package developed by a train manufacturer that gives us the required mechanical power when the train is in traction mode or the available power when the train is in regenerative braking mode. This model is good enough to test the reliability and the accuracy of the power flow method, but more complex train models may be developed.

\footnotetext{
${ }^{*}$ Corresponding author

Email addresses: arboleyapablo@uniovi.es (Pablo Arboleya), cotomanuel@uniovi.es (Manuel Coto), gonzalezmorcristina@uniovi.es (Cristina González-Morán), rarregui@caf .net (Ruth Arregui)
} 
In the present work, the authors develop a train model to be combined with the previous mentioned power flow approach. The proposed model combines the regenerative braking with an on-board accumulator, the so-called (ACR) developed by CAF Company. ACR is a Spanish acronym that stands for to Acumulador de Carga Rapida (Fast Charge Acummulator). The train can work under two different modes. The first one is the traction mode. In this mode, the required power can be provided by the catenary or the ACR depending on the ACR charge level, the amount of power demanded by the train and the catenary voltage. The second one is the braking mode, in which the power can be injected in the catenary, used to charge the ACR or burned in the rheostatic braking system depending on the operating variables. The squeezing control is also simulated when the catenary voltage exceeds a given value. Thus, part of the power is derived to the rheostatic system, and over a given catenary voltage value, no power can be injected in the catenary and all regenerated power must be burned. the developed model is a general parametric model that could be used with most of the accumulation technologies.

The authors must remark that this model was developed for power flow purposes, that is why the model do not include any derivative term considering the train dynamics or the electrical network dynamics. A more accurate formulation considering such dynamic behaviour will make the formulation much more difficult and it wont add more information or accuracy when calculating the power flow solution. The authors run a steady state simulations at each simulation instant neglecting the transients between successive instants as it was proposed in [8]. This approach, known as stationary equivalent method for moving loads, is widely accepted among the authors, not only for modelling DC traction networks, but also for modelling AC traction networks for high speed trains [2-5, 9-14]. This method assumes that the speed of the trains is not very high to induce pronounced electrical transients and the dc traction network slowly moves from one state to another as the locations and the input power of the trains vary. For this reason, steady state problems are solved at each instant, neglecting the electrical transients and the dynamic of the components. It is true that this is a simplification of the reality, and a differential model solved for transient purposes would be more accurate. However, it must be taken into account that these kind of software packages are designed for running during long time intervals in networks containing large number of trains, so very simple models solved with computationally efficient algorithms are required.

In the Section 2, the power flow equations are summarized and in Section 3 the train model is presented. Section 4 describes the solving procedure and in Section 5 the test results are presented.

\section{Power Flow Equations}

All linear equations are stated in a compact matrix form based in graph theory. The proposed method uses the node incidence matrix $\boldsymbol{\Gamma}$ to obtain such equations, simplifying the implementation procedure when compared with the traditional one [1-6]. A detailed description of this method can be found in [7]. The use of $\boldsymbol{\Gamma}$ for summarising the network topology is well known in graph theory [15]. The $\boldsymbol{\Gamma}$ rows and columns respectively represent the graph edges (lines or branches in the present case) and nodes (substations). The $\boldsymbol{\Gamma}_{i j}$ elements are defined as follows; $\left(\boldsymbol{\Gamma}_{i j}=1\right)$ when positive current in branch $i$, leaves node $j,\left(\boldsymbol{\Gamma}_{i j}=-1\right)$ when positive current in branch $i$, flows towards node $j$ and $\left(\boldsymbol{\Gamma}_{i j}=0\right)$ when no connection exists.

Using the $\boldsymbol{\Gamma}$ matrix, all equations representing Kirchhoff's Voltage and Current Laws, can be expressed in a compact form as follows:

$$
\mathbf{M z}^{T}=0
$$

where $\mathbf{z}$ is the vector representing voltages and currents in all network nodes and branches respectively and is constructed in the following form:

$$
\begin{aligned}
& \mathbf{z}=\left[\begin{array}{llllllll}
\mathbf{i}_{B}^{D C} & \mathbf{i}_{B d}^{A C} & \mathbf{i}_{B q}^{A C} & \mathbf{i}_{t}^{D C} & \mathbf{i}_{B}^{L} & \mathbf{i}_{B d}^{L} & \ldots
\end{array}\right. \\
& \left.\begin{array}{llllllll}
\ldots & \mathbf{i}_{N d}^{A C} & \mathbf{i}_{B q}^{L} & \mathbf{i}_{N q}^{A C} & \mathbf{v}_{N}^{D C} & \mathbf{v}_{N d}^{A C} & \mathbf{v}_{N q}^{A C} & ]
\end{array}\right]
\end{aligned}
$$

Where super-indices $D C, A C$ and $L$ represent magnitudes from the DC, AC and link subsystems respectively. Being the link subsystem the one that connects the AC and the DC subsystems. The sub-indices $B, t$ and $N$ represent branches, trains and nodes respectively. The variables of the AC subsystem are expressed in $d q$ components (subindices $d$ and $q$ represent direct and quadrature axis), and the variables of the DC subsystem are constant values. In the links, the variables are expressed in both $d q$ components and with a constant value.

The construction of $\mathbf{M}$ is represented in expression (3), where: 
- $\boldsymbol{\Gamma}^{D C}$ and $\boldsymbol{\Gamma}^{A C}$ represent the DC and AC subsystems topology respectively, defining a constant index for each line and each node. The AC topology will remain constant so $\Gamma^{A C}$ represents all real connections in the AC subsystem. However, $\boldsymbol{\Gamma}^{D C}$ represents all possible connections in the DC subsystem. For instance, a given train will be connected to all trains and all substations in the $\boldsymbol{\Gamma}^{D C}$. Then, only the actual connections will be activated for a given instant by means of $\mathbf{R}_{B}^{D C}$.

- $\mathbf{R}_{B}^{D C}$ is the branch resistance matrix of the DC subsystem. It is a diagonal matrix and $r_{i i}$ represents the resistance of branch $i$. As it was mentioned, $\boldsymbol{\Gamma}^{D C}$ generates a set of DC lines that are not active simultaneously at the same simulation step. The use of this formulation permits the authors to assign an infinite value to those inactive lines so they do not have any influence in the system. $\mathbf{R}_{B}^{D C}$ is the only matrix affected by the train motion, and must be updated when a train changes its location.

- $\mathbf{R}_{B}^{A C}$ and $\mathbf{X}_{B}^{A C}$ are the resistance and reactance matrices respectively, representing the impedance between AC nodes. They are diagonal matrices, where $r_{i i}$ and $x_{i i}$ represent the resistance and reactance of branch $i$ respectively, or the short circuit resistance and reactance of the transformer placed in the branch, in case of the AC branches that connect the AC and the DC system.

- $\mathbf{I}$ is the identity matrix.

- $\mathbf{S}$ is a block diagonal matrix. The first block is an identity matrix of dimensions $\left(n_{t}, n_{t}\right)$ and the second block is a diagonal matrix denoted as $\mathbf{S}_{\left(n_{s}, n_{s}\right)}^{L}$. Element $s_{i i}$ belonging to $\mathbf{S}^{L}$ is 1 if the DC substation $i$ is connected to the $\mathrm{AC}$ network, and 0 when the $\mathrm{DC}$ substation $i$ is not connected to the $\mathrm{AC}$ grid.

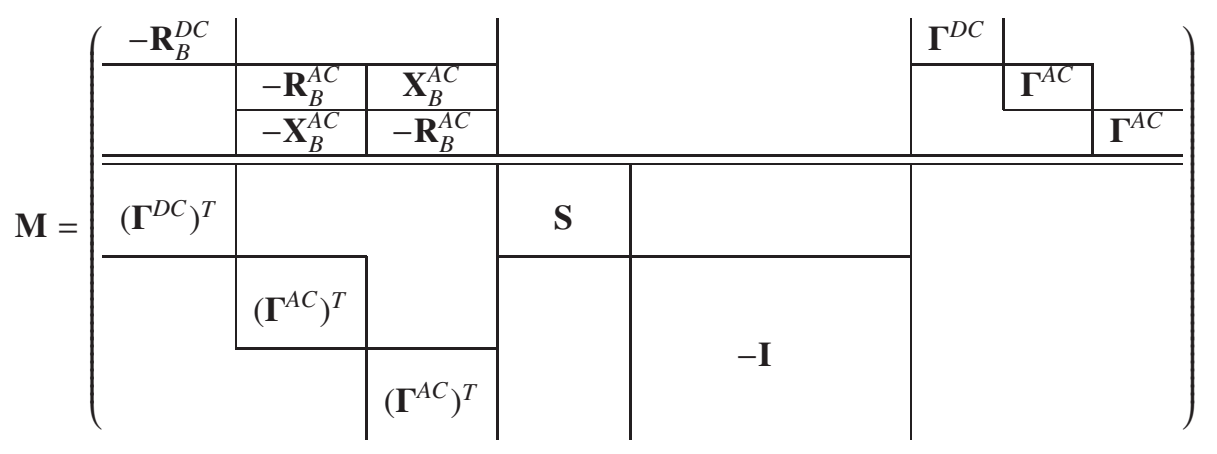

To complete the description of the system, the equations derived from the AC network, PQ and PV nodes, converters and trains must be added. $\mathrm{PQ}$ nodes in the $\mathrm{AC}$ network contribute with the next expressions:

$$
\left\{\begin{array}{c}
v_{N d i}^{A C} i_{N d i}^{A C}+v_{N q i}^{A C} i_{N q i}^{A C}-P_{i}=0 \\
v_{N q i}^{A C} i_{N d i}^{A C}-v_{N d i}^{A C} i_{N q i}^{A C}-Q_{i}=0
\end{array}\right.
$$

The equations corresponding to PV nodes are:

$$
\left\{\begin{array}{c}
v_{N d i}^{A C} i_{N d i}^{A C}+v_{N q i}^{A C} i_{N q i}^{A C}-P_{i}=0 \\
\sqrt{\left(v_{N d i}^{A C}\right)^{2}+\left(v_{N q i}^{A C}\right)^{2}}-\left|v_{N i}^{A C}\right|=0
\end{array}\right.
$$

The following equations correspond to a simple model of an AC/DC 6 pulse diode converter:

$$
\left\{\begin{array}{l}
v_{N d i}^{L} i_{B d i}^{L}+v_{N q i}^{L} i_{B q i}^{L}-v_{N i}^{L} i_{B i}^{L}=0 \\
v_{N q i}^{L} i_{B d i}^{L}-v_{N d i}^{L} i_{B q i}^{L}=0 \\
v_{N i}^{L}-1.35 \sqrt{\left(v_{N d i}^{L}\right)^{2}+\left(v_{N q i}^{L}\right)^{2}}-R_{e q i} i_{B i}^{L}=0 \\
3
\end{array}\right.
$$


Where $R_{\text {eqi }}$ is the equivalent resistance of the conversion unit in the regular commutation range. Further details can be obtained from $[16,17]$. Using the same procedure, complex models of non-controlled or controlled converters can be implemented.

As it was mentioned, when the construction of $\Gamma^{D C}$ and $R_{B}^{D C}$ was explained, the change of the location is already considered and can be set in a quite simple way using the procedure described in [7] without the need of changes in the main topology of the system. Traditional methods state a new power flow problem for each instant considering that the number of trains in the system and their relative position can vary [1-6]. Different instants might create different system topologies with different number of nodes and different connection between them. Applying the same enumeration criteria to two different instants, the same element (train or branch) can be labeled with different indices. This makes the comparison between different instants a very hard task. A tracking subroutine to search for a specific variable in the solution vector to be compared to the same variable at a different time instant must be developed.

Using the graph theory and the $\Gamma$ based formulation, the proposed method creates an invariant topology description even when the trains change their location or came in or out of the stage. The method asumes that any train that is present in the system during any instant of the simulation interval will be there for all instants, but it will be just activated when needed. The second assumption of the method is that all connections between trains and between trains and substations are considered. Regarding the connections between substations, only the real lines will be taken into account. Under these assumptions a case of study with two substations (N3, N4) and two trains (N1, N2) is represented in figure 1. In figure 1a), all possible connections between the trains are represented in dotted lines (for this specific case of study only B1), then all possible connections between the two trains and the two substations are represented in dashed lines (B2, B3, B4 and B5). Finally only the real connections between the two substations (B6 and B7) are represented in solid lines. Figures 1b) and c) represent two different scenarios with different train allocations. In the Scenario 1 (figure 1b)), the two trains are in line B7, the train 2 is near to the substation 3 and train one near the substation 4 . In this case the real line B7 is deactivated and replaced by the activation of branches B1, B3 and B4. There are no trains in line 6, so the real line 6 is active. Lines B2 and B5 representing the connection of train 1 with substation 3 and train 2 with substation 4 are deactivated too. In the scenario 2 depicted in the figure 1c) the train 1 is placed in the line B6 and the train 2 in the line B7, so these lines are deactivated. The branches connecting the train 1 with substations N3 and N4 (B2 and B3 respectively) are active as well as the branches connecting the train 2 with substations N3 and N4 (B4 and B5 respectively). Obviously in this case the branch B1 representing the connection between trains 1 and 2 is not activated.

No matter a branch is active or not, the matrix $\Gamma^{D C}$ is an invariant matrix. The activation or deactivation of the branches is carried out through the update of $R_{B}^{D C}$ at each instant. As it was mentioned earlier, this matrix is a diagonal matrix where each element represents the branch resistance. Those branches that are not active are set to a very high value of resistance. In this case, working in per unit system, the value assigned to the resistance of those non active branches is $10^{3}$ p.u. The resistance of the active branches changes as a function of the distance for the cases of connections between trains or between trains and substations. This is the case of B1 to B5. The branches representing the real connections (B6 and B7), have a fixed value if they are active. The procedure to deactivating a train is setting to $10^{3}$ p.u. the resistance of all branches connected to that train. For instance, in case of train N1 is deactivated, these branches would be B1, B2 and B3 (see figure 1a)).

With the described procedure the change in the train location is modelled by updating the diagonal matrix $R_{B}^{D C}$ at each instant, keeping constant the rest of the matrix $M$. This procedure makes really easy to update the train allocation and the comparison between different instants.

\section{Train Model}

The authors have used a complex software package developed by the train manufacturer CAF. This software calculates, for a given train and route, the available or demanded mechanical power in the machine when the train is in braking or traction mode depending on the rails and the train parameters. However, the train interactions with the DC power system, the ACR behaviour and amount of power burned in the rheostatic braking module depend on several factors such us the catenary voltage and ACR charge level. For this reason, to have a complete understanding of how the train works, a power flow problem must be solved taking into consideration not only the train under study, but also the traction network and other trains. 


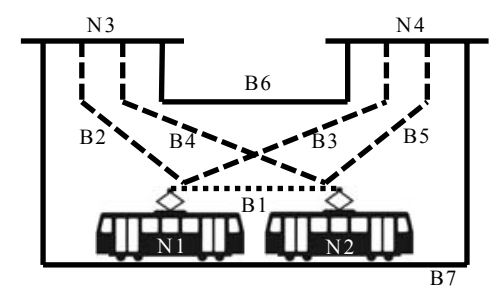

a)
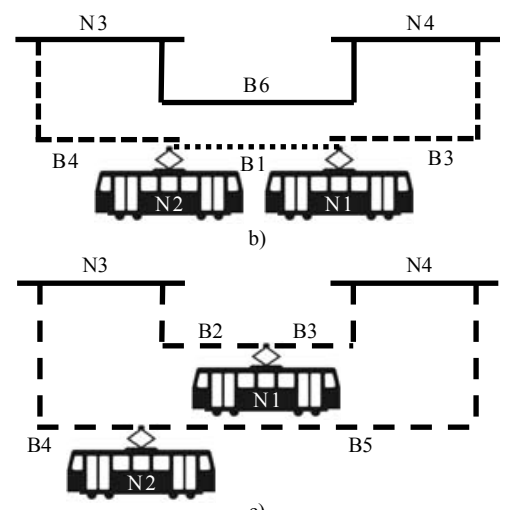

Figure 1. DC subsystem representation. a) General case considering all possible connections and locations of the trains. b) Scenario 1, with both trains located in line B7. Take notice that when at least one train is located in a real line (B7 in this case), the line is deactivated and substituted by other connections (B4, B1 and B3 in this case). c) Scenario 2 with train 1 located in line B6 and train 2 located in the line B7. In this case line B6 is deactivated and substituted by B2 and B3, and line B7 is deactivated too and substituted by B4 and B5.

\subsection{Traction mode}

The aim of this method is basically to obtain two values, the current that the train injects or absorbs from the catenary and the power injected to or absorbed from the ACR at each moment.

When the train is in traction mode, the maximum power that can be extracted from the ACR depends on its charge level. Usually, when the accumulator is being discharged near the minimum energy point, the maximum discharge power is reduced to extend the life of the system. A simple model could consider these functions as constant functions. In fact, if the systems do not check the accumulator level of charge to determine the power that can be extracted from it, this function would be a constant function.

$$
P_{\text {max }}^{A C R}=f_{\text {discharge }}\left(E^{A C R}\right)
$$

The authors would like to remark that this function is a general function and its description will vary from one manufacturer to another, in most of the cases is confidential. For the tests that explained in this paper the authors used a non-confidential version where the $P_{\max }^{A C R}$ is calculated as follows:

$$
P_{\text {max }}^{A C R}= \begin{cases}P_{\text {rated }}^{A C R} & E^{A C R} \geq k_{E} \cdot E_{\text {max }} \\ k_{P} \cdot P_{\text {max }}^{A C R} & E^{A C R} \leq k_{E} \cdot E_{\text {max }}\end{cases}
$$

Where $k_{P}$ and $k_{E}$ are numbers in the interval $(0,1)$. In the tests described in the paper $\left(K_{E}=0.3\right)$ and $\left(K_{P}=0.7\right)$.

However, even when the ACR charge level allows a given maximum power to be extracted from it $P_{\max }^{A C R}$, this quantity can be diminished for protection purposes if the catenary voltage is lower than a given value $\left(v_{t}^{A C R}\right)$. In (13), the available discharging power $P_{A v}^{A C R}$ is calculated as a function of the catenary voltage $v_{N i}^{D C}$.

$$
P_{A v}^{A C R}= \begin{cases}P_{\max }^{A C R} \cdot \frac{v_{N i}^{D C}}{V_{t}^{A C R}} & v_{N i}^{D C} \leq V_{t}^{A C R} \\ P_{\max }^{A C R} & v_{N i}^{D C} \geq V_{t}^{A C R}\end{cases}
$$


The traction system will need a given power at each instant $P_{r e f}$, that is calculated with the external software. Depending on whether this reference power is higher or not than the maximum power that can be extracted from the $\operatorname{ACR}\left(P_{\max }^{A C R}\right)$, the next casuistry will be developed for calculating the absorbed power from the catenary and the ACR, $P^{c a t}$ and $P^{A C R}$ :

- When $P_{\max }^{A C R} \geq P_{\text {ref }}$ :

$$
\begin{gathered}
P^{c a t}= \begin{cases}P_{r e f}-P_{\text {max }}^{A C R} \cdot \frac{v_{N i}^{D C}}{V_{t}^{A C R}} & v_{N i}^{D C} \leq V_{1} \\
0 & v_{N i}^{D C} \geq V_{1}\end{cases} \\
P^{A C R}= \begin{cases}P_{\max }^{A C R} \cdot \frac{v_{N i}^{D C}}{V_{t}^{A C R}} & v_{N i}^{D C} \leq V_{1} \\
P_{\text {ref }} & v_{N i}^{D C} \geq V_{1}\end{cases}
\end{gathered}
$$

- When $P_{\max }^{A C R} \leq P_{\text {ref }}$ :

$$
\begin{gathered}
P^{c a t}= \begin{cases}P_{\text {ref }}-P_{\max }^{A C R} \cdot \frac{v_{N i}^{D C}}{V_{t}^{A C R}} & v_{N i}^{D C} \leq V_{t}^{A C R} \\
P_{\text {ref }}-P_{\max }^{A C R} & v_{N i}^{D C} \geq V_{t}^{A C R}\end{cases} \\
P^{A C R}= \begin{cases}P_{\max }^{A C R} \cdot \frac{v_{N i}^{D C}}{V_{t}^{A C R}} & v_{N i}^{D C} \leq V_{t}^{A C R} \\
P_{\max }^{A C R} & v_{N i}^{D C} \geq V_{t}^{A C R}\end{cases}
\end{gathered}
$$

In figure 2 the above described model is represented. Where $V_{1}$ is the voltage when $P_{A v}^{A C R}$ reaches $P_{\text {ref }}$.

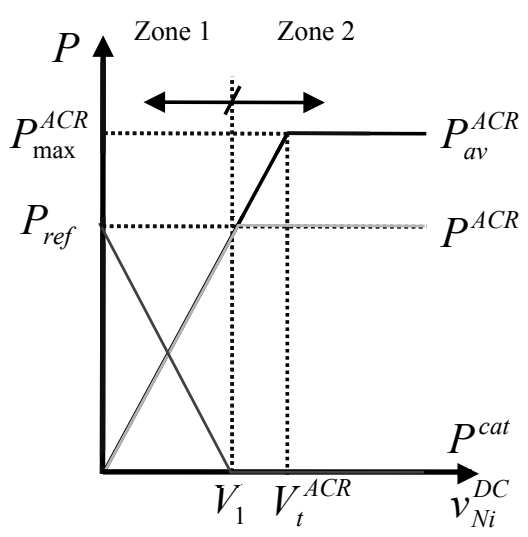

a)

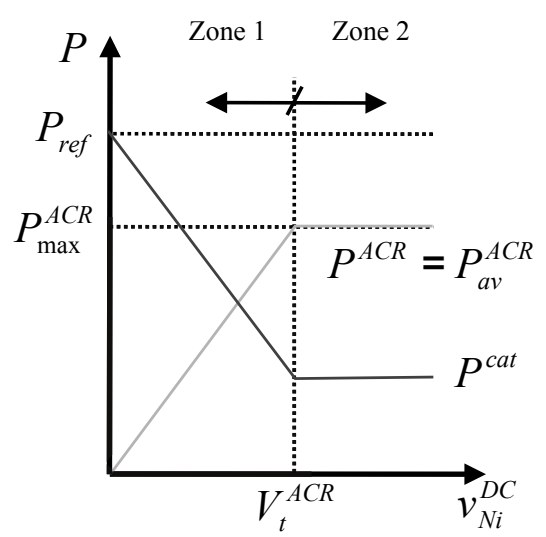

b)

Figure 2. a) Maximum power that ACR can develop is greater than the required electric traction power $\left(P_{\text {max }}^{A C R} \geq P_{\text {ref }}\right)$. b) Maximum power that ACR can develop is lower than the required electric traction power $\left(P_{\max }^{A C R} \leq P_{r e f}\right)$

In the final model current-voltage curves are used instead of the power-voltage previous ones, these current-voltage models are easily obtained considering that:

$$
\left\{\begin{array}{l}
P^{c a t}=v_{N i}^{D C} i_{t i}^{D C} \\
P^{A C R}=v_{N i}^{D C} i_{t i}^{A C R}
\end{array}\right.
$$

where $i_{t i}^{D C}$ and $i_{t i}^{A C R}$ are the absorbed currents from the catenary and the from ACR respectively and $v_{N i}^{D C}$ is the catenary voltage. From expressions (14)-(19) it can be stated: 
- When $P_{\max }^{A C R} \geq P_{\text {ref }}$ :

$$
\begin{gathered}
i_{t i}^{D C}= \begin{cases}\frac{P_{r e f}}{v_{N i}^{D C}}-\frac{P_{m a x}^{A C R}}{V_{t}^{A C R}} & v_{N i}^{D C} \leq V_{1} \\
0 & v_{N i}^{D C} \geq V_{1}\end{cases} \\
i_{t i}^{A C R}= \begin{cases}\frac{P_{\max }^{A C R}}{V_{t r e}} & v_{N i}^{D C} \leq V_{1} \\
\frac{P_{r e f}}{v_{N i}^{D C}} & v_{N i}^{D C} \geq V_{1}\end{cases}
\end{gathered}
$$

- When $P_{\max }^{A C R} \leq P_{\text {ref }}$ :

$$
\begin{gathered}
i_{t i}^{D C}= \begin{cases}\frac{P_{r e f}}{v_{N i}^{D C}}-\frac{P_{\max }^{A C R}}{V_{t}^{A C R}} & v_{N i}^{D C} \leq V_{t}^{A C R} \\
\frac{P_{r e f}-P_{\max }^{A C D}}{v_{N i}^{D C}} & v_{N i}^{D C} \geq V_{t}^{A C R}\end{cases} \\
i_{t i}^{A C R}= \begin{cases}\frac{P_{\max }^{A C R}}{V_{t}^{A C R}} & v_{N i}^{D C} \leq V_{t}^{A C R} \\
\frac{P_{\max }^{A C R}}{V_{N i}^{D C}} & v_{N i}^{D C} \geq V_{t}^{A C R}\end{cases}
\end{gathered}
$$

The piecewise defined functions have been implemented using the Fischer-Burmeister approach described in the Appendix in order to avoid the use of (if - them) implementation and increase the speed of the algorithm.

\subsection{Braking mode}

In this subsection, the authors will explain the interaction between the regenerative braking system, the rheostatic braking system, the ACR system and the DC traction network. First, it is explained how to model the train when it only has regenerative braking but no ACR system. Then the ACR model will be included. The same procedure could be applied to other systems, see for instance [18-20].

The characteristic that describes the train behaviour when it is working on regenerative braking mode can be observed in figure $3 \mathrm{a}$ and it is described in [21]. If the catenary voltage is lower than a given value $V_{0}$, the maximum injected current into the DC traction network is $I_{0}$. Above this voltage the squeezing control will act reducing the maximum value of the injected current. If the catenary voltage exceeds $V_{\max }$, then the squeezing control cuts off the current injection in the catenary. When not all the regenerated power can be injected in the catenary, the surplus is burned in the rheostatic braking system.

As it can be observed, there exist three zones defined by the following equations:

$$
i_{t i}^{D C}= \begin{cases}\frac{P_{r e f}}{v_{N i}^{D C}} & v_{N i}^{D C} \leq V_{c} \\ m\left(v_{N i}^{D C}-V_{\max }\right) & V_{c} \leq v_{N i}^{D C} \leq V_{\max } \\ 0 & v_{N i}^{D C} \geq V_{\max }\end{cases}
$$

Where $m=I_{0} /\left(V_{\max }-V_{0}\right)$ and $P_{\text {ref }}$ is the regenerated power. In Zone $1\left(v_{N i}^{D C} \leq V_{c}\right)$ all the regenerated power is injected in the network; $V_{c}$ is the cross point between $P_{\text {ref }}$ and squeezing control characteristic. In zone $2\left(V_{c} \leq v_{N i}^{D C} \leq\right.$ $\left.V_{\max }\right)$ the squeezing control starts, and part of the regenerated power is derived to the rheostatic braking system. In zone 3 all the regenerated power is burned in the rheostatic braking system. It should exist an extra zone where the current is limited to $I_{0}$ when the voltage is too low, for protection purposes. In this case, this zone has been avoided because in the simulations, such low voltages were never obtained.

In order to include the ACR system, it has to be considered that the maximum ACR charge power $P_{\max }^{A C R}$, depends on its charge level. This value can be diminished when the device is being charged for protection purposes if the catenary voltage is lower than a given value $\left(v_{t}^{A C R}\right)$ or if for instance the charge level of the accumulator is reaching its maximum. The aim of adding a complex function is to include in the model some protection systems for the accumulator or some procedures that will extend its life.

$$
P_{\max }^{A C R}=f_{\text {charge }}\left(E^{A C R}\right)
$$




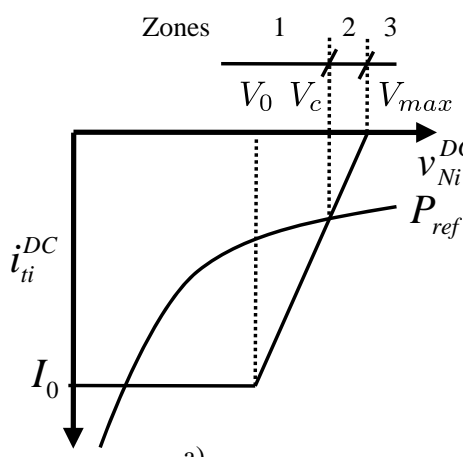

a)

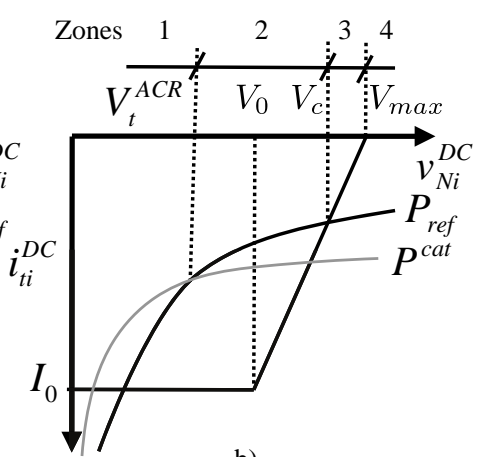

b)

Figure 3. a) Regenerative braking characterisit with no ACR system. b) Regenerative braking characterisit with ACR system.

If a simple system that does not check the level of charge of the accumulator or the catenary voltage is considered, this function could be a constant function. The actual functions used by the manufacturer can not be revealed in this paper, however, for the tests that are presented in the next sections the used function was the next:

$$
P_{\text {max }}^{A C R}= \begin{cases}P_{\text {rated }}^{A C R} & E^{A C R} \leq k_{E} \cdot E_{\text {max }} \\ k_{P} \cdot P_{\text {max }}^{A C R} & E^{A C R} \geq k_{E} \cdot E_{\text {max }}\end{cases}
$$

Again $k_{P}$ and $k_{E}$ are parameters between zero and one and for this particular case $\left(k_{E}=0.8\right)$ and $\left(k_{P}=0.8\right)$.

Figure 2 can also be used to analyze the performance of the system when the train is braking and the ACR is in charging mode. But in this case the sign of the powers should be inverted.

Combining the curves in figure 2 with the braking characteristic described in figure $3 \mathrm{~b}$, two different cases are obtained:

- When $\left|P_{\text {max }}^{A C R}\right| \geq\left|P_{\text {ref }}\right|$, all regenerated power can be used to charge the ACR except when the voltage is too low. In this case part of the regenerated power is injected into the DC traction system. It is important to take notice that $\left(V_{t}^{A C R} \leq V_{0}\right),\left(V_{c} \geq V_{0}\right)$ and $\left(V_{1} \leq V_{t}^{A C R}\right)$ so $\left(V_{1} \leq V_{c}\right)$ and only two Sections are needed to define the current injected in the network and the current injected in the ACR as it is expressed in the next equations:

$$
\begin{gathered}
i_{t i}^{D C}= \begin{cases}\frac{P_{r e f}}{v_{N i}^{D C}}-\frac{P_{m a x}^{A C R}}{V_{t}^{A C R}} & v_{N i}^{D C} \leq V_{1} \\
0 & v_{N i}^{D C} \geq V_{1}\end{cases} \\
i_{t i}^{A C R}= \begin{cases}\frac{P_{m a x}^{A C R}}{V_{t}^{A C R}} & v_{N i}^{D C} \leq V_{1} \\
\frac{P_{r e f}}{v_{N i}^{D C}} & v_{N i}^{D C} \geq V_{1}\end{cases}
\end{gathered}
$$

- When $\left|P_{\max }^{A C R}\right| \leq\left|P_{\text {ref }}\right|$ four zones are obtained. The final braking characteristics considering the regenerative braking system, the squeezing control and the ACR system are mathematically described in expressions (29) and (30). In zone 1 the ACR can not be charged using the maximum charging power so most of the energy is injected in the catenary. In zone 2 the ACR can be charged using $P_{\max }^{A C R}$, but in this case the regenerated power is bigger so part of the regenerated power is injected in the catenary. In zone 3 the squeezing control starts working and the injected power in the catenary is limited, so the rheostatic braking system starts working. In this case part of the power is used for charging the ACR system, other part is injected in the catenary and the last part is burned in the rheostatic system. Finally, in zone 4 no power can be injected in the catenary so part of the regenerated power is used for charging the ACR and the other part is burned in the rheostatic braking system. 


$$
\begin{aligned}
& i_{t i}^{D C}= \begin{cases}\frac{P_{r e f}}{v_{N i}^{D C}}-\frac{P_{\max }^{A C R}}{V_{\text {mat }}^{A C R}} & v_{N i}^{D C} \leq V_{t}^{A C R} \\
\frac{P_{r e f}-P_{\max }^{A C R}}{v_{N i}^{D C}} & V_{t}^{A C R} \leq v_{N i}^{D C} \leq V_{c} \\
m\left(v_{N i}^{D C}-V_{\max }\right) & V_{c} \leq v_{N i}^{D C} \leq V_{\max } \\
0 & v_{N i}^{D C} \geq V_{\max }\end{cases}
\end{aligned}
$$

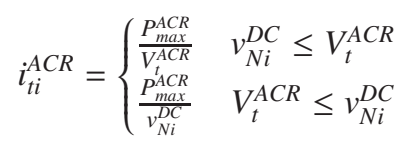

Like in the traction mode, in the braking mode, the piecewise defined functions have been implemented using the Fischer-Burmeister approach described in the Appendix in order to avoid the use of (if - them) implementation and increase the speed of the algorithm.

\section{Solving Procedure}

For a given simulation instant, the ACR charge level must be initialized. Then, depending on whether the train is in traction or in braking mode, expressions (11) or (25) are respectively used to calculate the maximum discharge or charge capability of the ACR. If the train is in traction mode, the maximum discharge capability $P_{\max }^{A C R}$ is compared with the demanded power $P_{r e f}$, and the characteristics $v_{N i}^{D C} / i_{t i}^{D C}$ and $v_{N i}^{D C} / i_{t i}^{A C R}$ are constructed following the above described procedure. The first one is used for solving the AC/DC unified power flow and the second one for updating the energy stored in the ACR system for the next iteration once the power flow is solved. The procedure followed when the train is in braking mode is quite similar. The maximum charge capability $P_{\max }^{A C R}$ is calculated as a function of the energy stored and compared with the available regenerated power. Then the $v_{N i}^{D C} / i_{t i}^{D C}$ and $v_{N i}^{D C} / i_{t i}^{A C R}$ characteristics are constructed and used in the power flow algorithm and in the ACR charge level updating respectively.

For validating the obtained results, the models have been implemented in MATLAB/SIMULINK considering static networks. The models followed the above described equations, but no change in the train location was simulated. Using this procedure and changing the power reference of the trains, a number of instants was tested and validated. The tests were quite extensive and were designed to cover all operating points of the model. The tests considered cases with a single train and cases with multiple trains. Once the models were validated using this procedure, they were embedded in the developed software package and sent them to the company to be validated using comparisons with real measured cases.

\section{Results}

Two cases of study will be presented in this Section. The first one, represents a single train through a single line system. The obtained results can be observed in figure 4. The initial energy level of the ACR is set to zero. Because of that, during the first instants of simulation, when the train is in traction mode, all the power is taken from the catenary and the voltage drop in the catenary is quite important. Then, when the train brakes, the ACR is charged. The energy stored in the ACR will support the network in further instants when the train is in traction mode. For instance, see the instants between 50s and 100s. The ACR is charged and the train starts a traction cycle, the energy stored in the ACR is reduced as it can be observed in figure $4 \mathrm{~d}$, and the voltage drop in the catenary is reduced comparing with the first instants when the ACR had no charge.

The second case of study represents a fictitious but quite common linear configuration with 10 stops, two of them connected to the AC network through rectifier substations. In this case all input data and network parameters were proposed by the University team to check the performance of the algorithm. The scheme of the studied configuration is represented in figure 5. As it can be observed, the linear network has 10 stops, and in two of them (2 and 6), the DC circuit is fed by the AC network using a rectifier substation. Both transformers are 22000/930V three-phase power transformers and the rectifiers are non controlled type. The rated voltage in the DC network is $1250 \mathrm{~V}$ and the maximum catenary voltage is set to $1500 \mathrm{~V}$. In this case $7.5 \mathrm{~min}$ are simulated with four trains in the network. The trains 1 and 3 depart from the stop 1 with destination stop 10 at $t_{1}=0 \mathrm{~min}$ and $t_{3}=1 \mathrm{~min}$ respectively. In the other 

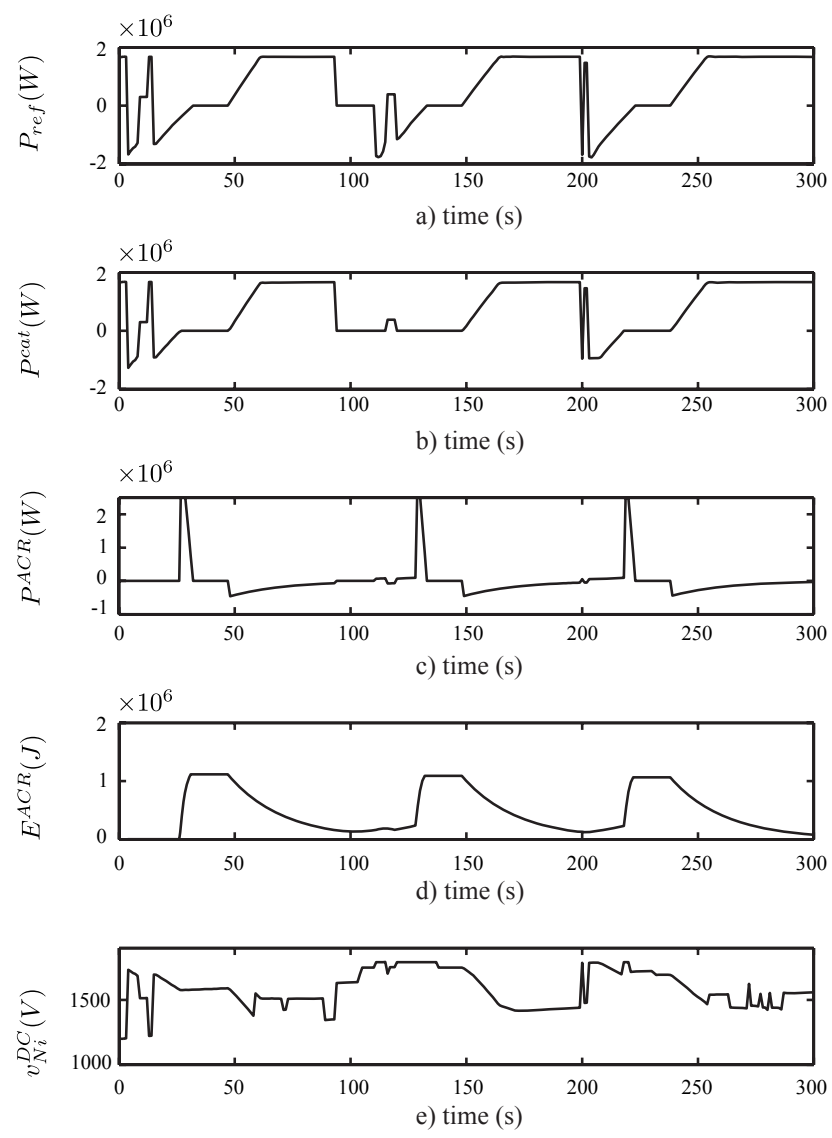

Figure 4. Results for the single train case. a) $P_{r e f}$ b) $P^{c a t}$ c) $P^{A C R}$ d) $E^{A C R}$ e) $v_{N i}^{D C}$

side, the trains 2 and 4 depart from the stop 10 with destination stop 1 at $t_{2}=1.8 \mathrm{~min}$ and $t_{4}=3.4$ min respectively. Obviously, the whole system is a dual track railway. For this case of study, two different scenarios will be considered, the first one assumes that all trains are conventional trains, they have regenerative braking system but not accumulator system. In the second scenario, all the trains are equipped with the accumulation system. The effect of the adding accumulation systems to the trains over the whole system will be evaluated.

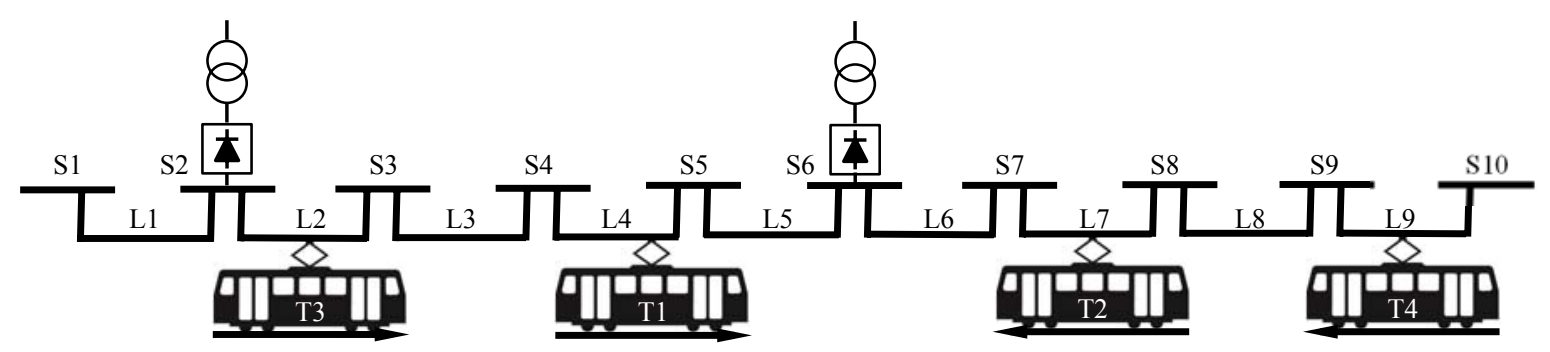

Figure 5. Scheme of the studied network for the second case of study

One of the most important parameters is the energy saving computation derived from the use of the accumulation system. In figure 6, the accumulated energy demand in both substations located at stops 2 and 6 is globally displayed. As it can be observed, during this short time interval of study of $7.5 \mathrm{~min}$ in a network with just 4 trains, the energy saving due to the accumulation system is about $12.72 \mathrm{kWh}$, that represents more than $11 \%$ of the total energy consumption. Obviously, the energy saving will depend on the number of trains and their behaviour, but as it can be 
observed, even for small networks with not many trains, the achieved saving is not negligible.

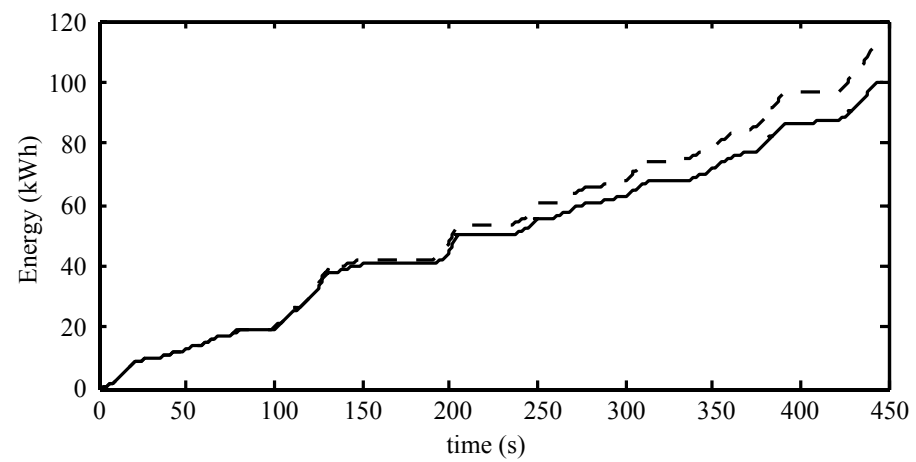

Figure 6. Total accumulated energy consumption measured at substation level. The solid line represent the second scenario, where all the trains are equipped with the accumulation system while the dashed line represents the scenario where the trains just have regenerative braking.

In figure 7 the evolution of the power demanded from both substations and the voltage in the DC side of such substations is represented. As it can be observed, the voltage profile is very similar in both substations when the case with accumulation system is compared with the conventional one. Most of the times when a significant difference arises, the case with the accumulation system in the trains (solid line), gives lower voltage peaks. This is because the charging of the accumulation systems limits the power injected in the network and thus, the voltage peak at the substations is also reduced.
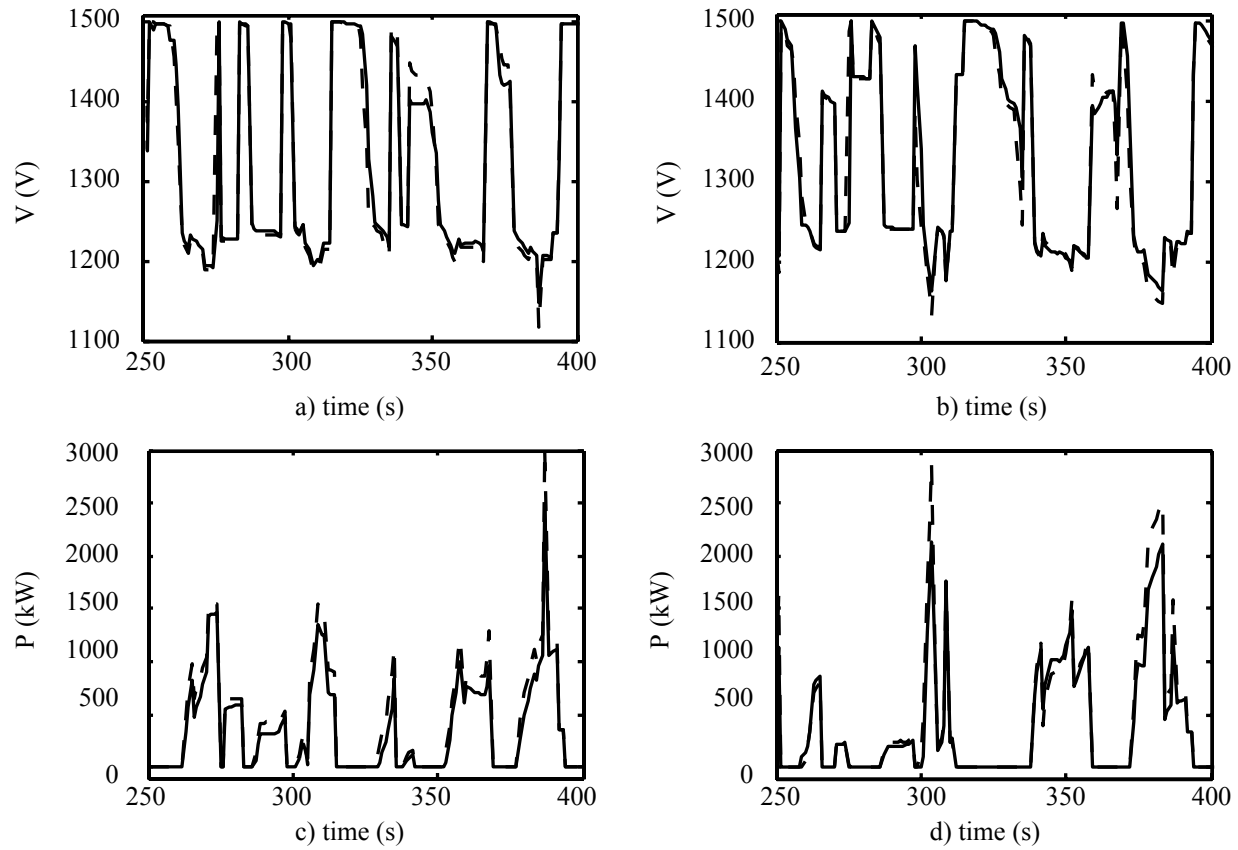

Figure 7. Power demanded from substations 1 and 2 and voltage in the DC side of the mentioned substations. The subfigures a) and c) represents respectively the voltage and the power at substation 1 located at stop 2, and subfigures b) and d) represents respectively the voltage and the power at substation 2 located at stop 6 . The solid line represent the scenario with accumulation system and the dashed line represents the scenario with convenitional trains.

Regarding the power demanded from the substations, the case with the accumulation system clearly requires a lower power demand, specially in the instants when a peak of power demand is produced. As it can be observed, the peak power demand is reduced from $3000 \mathrm{~kW}$ to nearly $2000 \mathrm{~kW}$, so in this case the use of the accumulation system 
could allow us a reduction in the size of the power transformers and rectifiers of the substations, or an increase of the traffic in the traction network using the same infrastructure. It has been only represented a time interval of $150 \mathrm{~s}$ (from 250 s to 400 s), because the analysis of the figures is easier and also this interval is the most critical because all the trains have departed which means that all of them are active.

As it can be observed, there are multiple instants where the demanded power at the substations is zero, this is derived from the effect of the regenerative braking of the trains, the regenerated power by trains in braking mode is used to feed trains in traction mode. In those situations, there is no energy flowing from the AC system to the DC subsystem. It is important to notice that in such situations, the voltage is very high, near the maximum, and this is a good indicator to know that there is a significant amount of regenerative power being injected by the trains in the DC network.

The analysis of the voltage at the other stops does not have any interest because these electrical nodes are not connected to the $\mathrm{AC}$ network and due to the train controls, the voltage values will be constrained between the maximum and the minimum allowed by the network operator.

In figure 8 , the train behaviour can be analysed between the instants $250 \mathrm{~s}$ and $400 \mathrm{~s}$. The figure is structured in matrix of $4 \times 4$ subfigures. Each column represents one of the four trains studied in this case. In the rows, the power reference representing the total demanded power or the power available for regenerating purposes, the actual power demanded or injected by the train in the catenary, the catenary voltage and the power and energy stored in the accumulation system are respectively depicted. Regarding rows 2 and 3, in solid line the scenario with accumulation system in the trains is represented, while the dashed lines represent the second scenario, when the trains do not have accumulation system.

Comparing the first row with the second row (Power reference and Power extracted or injected in the catenary), it can be observed that when the trains do not have accumulation system and the power reference is positive (traction mode), the power demanded from the catenary and the power reference are equal. In this situation all the power demanded by the traction system of the train will be extracted from the catenary. Take notice that during the interval of study the catenary voltage drop during the acceleration process is not high enough to activate the current limitation. In such case the power extracted from the catenary will be lower than the reference and the train traction system will not receive all the requested power.

When the trains are equipped with the accumulation systems, the peak power during the acceleration process is lower, because part of the power is provided by the accumulation system. Of course, if during the acceleration process there is no charge in the accumulation system, all the power will be demanded from the catenary. In those cases, the peak power with and without accumulation system will be the same. In row 4, the accumulation system stored energy is represented with solid line, and the power used to charge and discharge such system is depicted in dash-dotted line. It can be seen that the level of charge of the accumulation system in the train 3 is quite low, that is why the profile of the reference power the demanded energy from the catenary are nearly the same.

During the braking process (negative values in row 2 subfigures), when the trains are equipped with the accumulation system, the injected power in the catenary is lower, because part of the regenerated power is used to charge the accumulator. When the accumulator is full charged the injected power in the catenary is the same in both cases (with and without accumulator), see for instance train 4 at $\mathrm{t}=350 \mathrm{~s}$.

Regarding the voltage, the influence of the power injection in the catenary voltage, analysing the second and the third row it can be seen, that when the train injects a high amount of power in the catenary, the voltage increases. During the traction process, the voltage is lower. The voltage profiles comparing the case with and without accumulation are quite similar, some times the voltage peaks are shaved when there is accumulation system, this is because part of the regenerated power is not injected in the catenary because is being used to charge the ACR.

Finally, a report about the computational efficiency of the software package will be provided. The system of equations at all instants has been solved using the well-known trust-region dogleg algorithm [22]. The above described multi-train simulation was done using a conventional personal computer with a processor Intel(R) Core(TM) i72670QM CPU @ 2.20GHz and 4GB of RAM.

With the histogram depicted in figure 9 a comparison between the speed and efficiency of the algorithm and the number of iterations with and without the accumulation system can be carried out. During the interval of study ( $7.5 \mathrm{~s})$, 450 power flows where solved for both scenarios (with and without ACR). In the first case (without ACR) the whole simulation took $274 \mathrm{~s}$, while in the second case (with the ACR) the time invested for solving the whole interval was a longer (323 s). The use of the ACR produces an increasing in the computational time of $18 \%$ approximately. It 

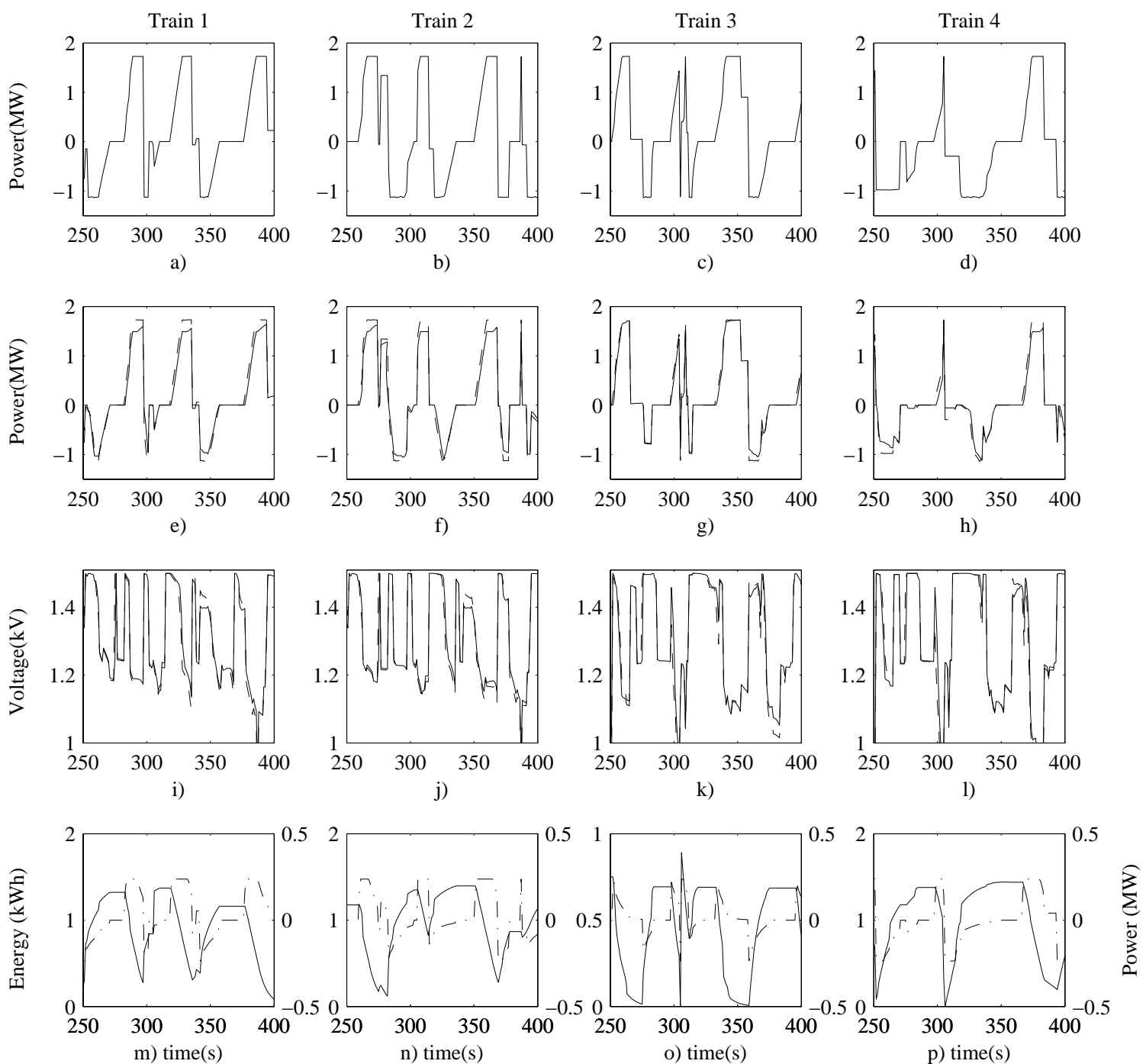

Figure 8. Study of the trains behaviour and their effect over the network voltage. This figure is structured in a $4 \times 4$ matrix of subfigures. Each column represents a train. In the first row of subfigures ( a), b), c) and d)), the reference power is represented for all trains, the reference power represents the power demanded by the traction system of the train when is in traction mode (positive) or the available power for being regenerated when the train is in braking mode (negative). The second row of subfigures (e), f), g) and h)) represents the the actual power demanded by the train in the catenary when it is in traction mode (positive) or injected by the train in the catenary when it is in braking mode (negative). In solid line the second scenario, when the trains are equiped with the accumulation systems is represented. The dashed line represents the first scenario when the trains just have a convenitional regenerative system. The third row of subfigures (i), j), k) and l) ) represents the catenary voltage in both scenarios. The solid line is used for representing the catenary voltages of the trains with the accumulation systems and the dashed line is used when the trains do not have accumulation system. Finally, in the fourth row of subfigures (m), n), o) and p), the power used for charging the accumulator (negative) or discharging it (positive) is represented in dashed-doted line, and the energy stored in the accumulator is represented in solid line. Obviously, this last row only represents the second scenario, when the trains are equipped with accumulators.

must be stated that solving this time interval with accumulation system using the piecewise definition of the functions was inviable due to the convergency problems derived from the iterative process among the different parts of the 
piecewise defined models. The mean time invested in solving each simulation step is $0.61 s$ in case of trains without accumulation, and $0.72 s$ in case the trains are equipped with the accumulation system (see figure 9 a) and c)). The mean number of iterations when the trains are equipped with accumulation system is 9.51 , while the same parameter when conventional trains are simulated is 8.33 (see figure $9 \mathrm{~b}$ ) and d)). As it can be observed in figure 9 the standard deviation of the number of iterations for solving each simulation step and the time invested are similar when the scenarios with and without accumulation are compared.
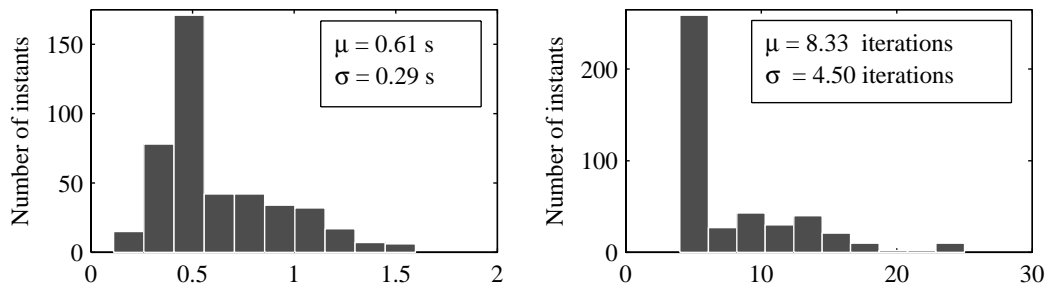

a) Computation time (without ACR model)

b) Number of iterations (without ACR model)
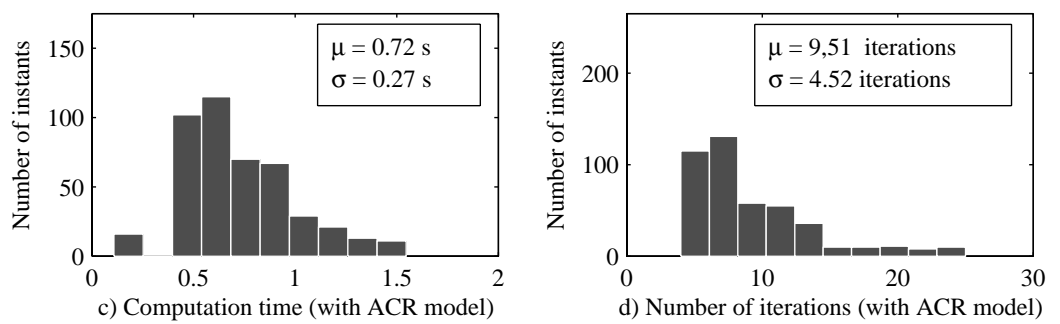

Figure 9. Computational efficiency of the software package evaluation. a) Histogram representing the computation time for each simulation step in the scenario 1 (trains without accumulation). b) Histogram representing the number of iterations invested for solving each time step in scenario 1 (trains without accumulation). c) Histogram representing the computation time for each simulation step in the scenario 2 (trains with accumulation). d) Histogram representing the number of iterations invested for solving each time step in scenario 2 (trains with accumulation). In each subfigure the mean value and the standard deviation of the studied variable are provided.

\section{Conclusion}

In the present work a model of a DC train with a regenerative braking system, a rheostatic braking system and an on-board fast charge accumulator (ACR) was presented for being applied in AC/DC unified power flow studies. The authors have developed a really simple model based on the construction of two single characteristics when the train is in traction mode and another two when the train is in braking mode. The train characteristics must be updated at each iteration because they depend on the ACR charge level, (that is updated at each iteration too), and the demanded or regenerated power, when the train is in traction mode or in braking mode respectively. It have been demonstrated that the developed model of the train with on board accumulator and regenerative braking system can be embedded in a software package that models the trains interactions with the electrical network using a power flow approach.

\section{Appendix}

To increase the efficiency of the algorithm, the authors have reformulated the problem as a nonlinear complementary problem (NCP). In [23] a detailed explanation of this method is given, but summarising, it could be stated that in the case under study, the value of $i_{t i}^{D C}$ changes abruptly depending on the value of $v_{N i}^{D C}$ that is a solution of the power flow problem.

Assuming for instance that $P_{\max }^{A C R} \geq P_{\text {ref }}$, it can be observed in equation (20), that one expression must be chosen if $V_{N i}^{D C} \leq V_{1}$ and another expression if $V_{N i}^{D C} \geq V_{1}$, so in $V_{1}$ the value of $i_{t i}^{D C}$ will suffer a brusque change. It means that the system is represented by two different models depending on the value of the solution $V_{N i}^{D C}$, so this method requires 
continuous checking at each iteration of the solution to verify if the chosen model was the right one. This represents a serious disadvantage to solve the problem using Newton-Raphson based algorithms (see [24] Sec.7 and [23]).

In this paper, each time that two different models or functions are used to represent a device, the two different models are remapped using NCP-functions and they are merged in a unique function model avoiding the need of making a choice. It must be pointed out that with this remapping, there non-linearity issue is not remove at all, it is just transformed the way in which the non-linearity is expressed, the problem is exactly the same before and after replacing the piecewise defined functions by the new NCP ones. The authors do not transform a non-linear problem into a linear one, but this procedure is fastest than the (if - them) procedure even when the Jacobian matrix must be recomputed at each iteration, as it occurs with the conventional (if - them) based procedure for piecewise defined functions.

A NCP-function can be defined as a function $\Phi: \mathfrak{R}^{2} \rightarrow \mathfrak{R}$ that satisfies [23, 25]:

$$
\Phi(x, y)=0 \Leftrightarrow x \geq 0, y \geq 0, x y=0
$$

This function is zero if at least on of the inputs $x$ or $y$ is zero. Among the several NCP-functions available [25-27] the authors opted, like in [23, 28], for Fischer-Burmeister functions [29].

$$
\Phi_{F B}(x, y)=\sqrt{x^{2}+y^{2}}-(x+y)
$$

If two different functions $F_{1}$ and $G_{1}$ are defined using the model expressed in (20) such us:

$$
\begin{gathered}
F_{1}\left(v_{N i}^{D C}\right)=i_{t i}^{D C}-\frac{P_{r e f}}{v_{N i}^{D C}}-\frac{P_{m a x}^{A C R}}{V_{t}^{A C R}} \\
G_{1}\left(v_{N i}^{D C}\right)=i_{t i}^{D C}
\end{gathered}
$$

Then, a Fischer-Burmeister function can be built as follows:

$$
\Phi_{F B}\left(F_{1}, G_{1}\right)=\sqrt{F_{1}^{2}\left(v_{N i}^{D C}\right)+G_{1}^{2}\left(v_{N i}^{D C}\right)}-F_{1}\left(v_{N i}^{D C}\right)-G_{1}\left(v_{N i}^{D C}\right)=0
$$

Then, this function will be zero if at least one of the inputs $F_{1}$ or $G_{1}$ is zero, which actually means that equations in (20) can be substituted by the equation (A.5). Making the equation (A.5) equal to zero means one of these statements:

- The first model is activated.

- The second model is activated.

- Both models are activated at the same time at point $V_{1}$ (see figure 2). It must be remarked that in this case there is no need of calculating $V_{1}$ because it is implicit in the equation (A.5).

In figures A.1 and A.2, the obtained smooth traction characteristics using the mentioned approach for two cases of study are represented.

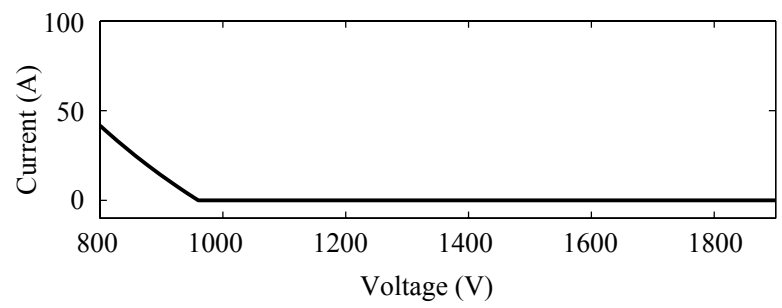

Figure A.1. Traction characteristic $i_{t i}^{D C} / v_{N i}^{D C}$ when $\left(P_{\max }^{A C R} \geq P_{r e f}\right) . P_{r e f}=200 k W, P_{\max }^{A C R}=250 \mathrm{~kW}, V_{t}^{A C R}=1200 \mathrm{~V}$. 


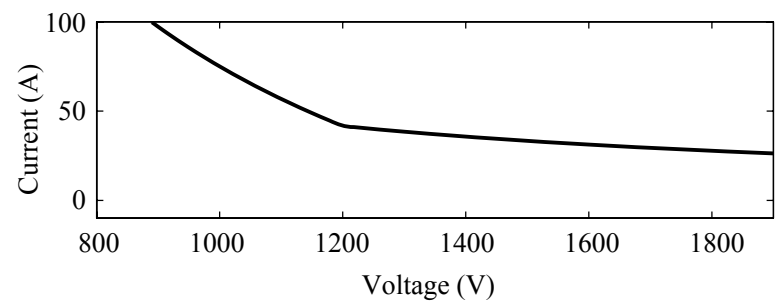

Figure A.2. Traction characteristic $i_{t i}^{D C} / v_{N i}^{D C}$ when $\left(P_{\max }^{A C R} \leq P_{\text {ref }}\right) . P_{\text {ref }}=200 k W, P_{\max }^{A C R}=150 \mathrm{~kW}, V_{t}^{A C R}=1200 \mathrm{~V}$.

\section{References}

[1] M. C. Falvo, R. Lamedica, R. Bartoni, G. Maranzano, Energy management in metro-transit systems: An innovative proposal toward an integrated and sustainable urban mobility system including plug-in electric vehicles, Electric Power Systems Research 81 (12) (2011) 2127 2138 .

[2] Y. Tzeng, R. Wu, N. Chen, Electric network solutions of dc transit systems with inverting substations, Vehicular Technology, IEEE Transactions on 47 (4) (1998) 1405-1412.

[3] Y. Tzeng, R. Wu, N. Chen, Unified ac/dc power flow for system simulation in dc electrified transit railways, Electric Power Applications, IEE Proceedings 142 (6) (1995) 345-354.

[4] C. Pires, S. Nabeta, J. Cardoso, Dc traction load flow including ac distribution network, Electric Power Applications, IET 3 (4) (2009) 289-297.

[5] D. Braunagel, L. Kraft, J. Whysong, Inclusion of dc converter and transmission equations directly in a newton power flow, Power Apparatus and Systems, IEEE Transactions on 95 (1) (1976) 76-88.

[6] T. Smed, G. Andersson, G. Sheble, L. Grigsby, A new approach to ac/dc power flow, Power Systems, IEEE Transactions on 6 (3) (1991) $1238-1244$

[7] P. Arboleya, G. Diaz, M. Coto, Unified ac/dc power flow for traction systems: A new concept, Vehicular Technology, IEEE Transactions on 61 (6) (2012) 2421-2430.

[8] S. Talukdar, R. Koo, The analysis of electrified ground transportation networks, Power Apparatus and Systems, IEEE Transactions on 96 (1) (1977) 240-247.

[9] C. Pires, S. Nabeta, J. Cardoso, Iccg method applied to solve dc traction load flow including earthing models, Electric Power Applications, IET 1 (2) (2007) 193-198.

[10] L. Abrahamsson, L. Soder, Fast estimation of relations between aggregated train power system data and traffic performance, Vehicular Technology, IEEE Transactions on 60 (1) (2011) 16-29.

[11] Y. Cai, M. Irving, S. Case, Iterative techniques for the solution of complex dc-rail-traction systems including regenerative braking, Generation, Transmission and Distribution, IEE Proceedings 142 (5) (1995) 445-452.

[12] E. Pilo, R. Rouco, A. Fernandez, A. Hernandez-Velilla, A simulation tool for the design of the electrical supply system of high-speed railway lines, in: Power Engineering Society Summer Meeting, 2000. IEEE, Vol. 2, 2000, pp. 1053-1058 vol. 2.

[13] E. Pilo, R. Rouco, A. Fernandez, A reduced representation of 2 times; 25 kv electrical systems for high-speed railways, in: Rail Conference, 2003. Proceedings of the 2003 IEEE/ASME Joint, 2003, pp. 199-205.

[14] M. Coto, P. Arboleya, C. Gonzalez-Moran, Optimization approach to unified ac/dc power flow applied to traction systems with catenary voltage constraints, International Journal of Electrical Power \& Energy Systems 53 (0) (2013) 434 - 441.

[15] P. Murty, Power systems analysis, BSP, 2009.

[16] P. Pozzobon, Transient and steady-state short-circuit currents in rectifiers for dc traction supply, Vehicular Technology, IEEE Transactions on 47 (4) (1998) 1390-1404.

[17] F. Milano, Power System Modelling and Scripting, Springer, 2010.

[18] K. Divya, J. stergaard, Battery energy storage technology for power systemsan overview, Electric Power Systems Research 79 (4) (2009) 511 -520 .

[19] S. Danielsen, O. B. Fosso, M. Molinas, J. A. Suul, T. Toftevaag, Simplified models of a single-phase power electronic inverter for railway power system stability analysisdevelopment and evaluation, Electric Power Systems Research 80 (2) (2010) $204-214$.

[20] A. D. Bernardinis, S. Butterbach, R. Lallemand, A. Jeunesse, G. Coquery, P. Aubin, Double resonant isolated converter for battery charger with fast switching semiconductors used in hybrid electric shunting locomotive, Electric Power Systems Research 92 (0) (2012) 43 - 49.

[21] Y. Okada, T. Koseki, K. Hisatomi, Power management control in dc-electrified railways for the regenerative braking systems of electric trains, Advances in Transport 15 (2004) 919-929.

[22] T. Steihaug, The conjugate gradient method and trust regions in large scale optimization, SIAM Journal on Numerical Analysis 20 (3) (1983) $626-637$.

[23] G. Diaz, C. Gonzalez-Moran, Fischer-burmeister-based method for calculating equilibrium points of droop-regulated microgrids, Power Systems, IEEE Transactions on 27 (2) (2012) 959-967.

[24] F. Facchinei, J. Soares, A new merit function for nonlinear complementarity problems and a related algorithm, SIAM Journal on Optimization 7 (1) (1997) 225-247.

[25] P. M. Pardalos, T. M. Rassias, A. A. Khan, Nonlinear Analysis and Variational Problems: In Honor of George Isac, Springer, 2010.

[26] S. Dempe, V. V. Kalashnikov, Optimization With Multivalued Map- pings: Theory, Applications, and Algorithms, Springer, 2010.

[27] D. Sun, L. Qi, On ncp-functions, Computational Optimization and Applications 13 (1-3) (1999) 201-220. 
[28] M. Coto, Unified power flow (ac/dc) applied to traction networks, Ph.D. thesis, University of Oviedo, Spain (2012).

[29] A. Fischer, A special newton-type optimization method, A Journal of Mathematical Programming and Operations Research 24 (1992) 269284. 